\title{
Interactive comment on "High-resolution Hybrid Inversion of IASI Ammonia Columns to Constrain U.S. Ammonia Emissions Using the CMAQ Adjoint Model" by Yilin Chen et al.
}

Yilin Chen et al.

chenylis10@gmail.com

Received and published: 3 January 2021

We thank the reviewer for providing insightful comments. Please see the supplement for detailed responses.

Please also note the supplement to this comment:

https://acp.copernicus.org/preprints/acp-2020-523/acp-2020-523-AC1-supplement.pdf 